\title{
Clinical evaluation on the determination of plasma lysophosphatidic acid concentration in Chinese human pancreatic cancer
}

\author{
Gong Yongling, Wang Shaokai, Tao Chenjie, Chen Jinfei, \\ Wang Shukui, Cao Xiufeng, Lv Guangmei, Li Pin
}

\begin{abstract}
Aims: Observing the alteration of plasma Lysophosphatidic acid (LPA) concentration in patients with pancreatic cancer and evaluating its clinical potential for diagnosis. Methods: We examined the plasma LPA concentrations by using a LPA assay kit and related parameters of CA19-9, AFP and CEA were measured in 50 patients with pancreatic cancer, 32 patients with benign pancreatic lesions and 36 healthy checkup volunteers. Findings were analyzed and correlation with pathological changes. Results: The LPA concentration was significantly higher in pancreatic cancer patients $(4.10 \pm 2.03$ $\mu \mathrm{mol} / \mathrm{L}$ ) than in patients with benign lesions $(3.28 \pm 1.26 \mu \mathrm{mol} / \mathrm{L})$ and healthy controls $(2.27 \pm 1.02 \mu \mathrm{mol} / \mathrm{L})(\mathrm{p}<0.05)$. Plasma LPA concentration correlated with serum CA19-9 level $(r=0.9070)$ in patients with pancreatic cancer. For diagnosis of pancreatic cancer, the sensitivity of LPA was $89.6 \%$ and the specificity
\end{abstract}

Gong Yongling ${ }^{1}$, Wang Shaokai ${ }^{1}$, Tao Chenjie ${ }^{1}$, Chen Jinfei $^{1}$, Wang Shukui ${ }^{2}$, Cao Xiufeng ${ }^{1}$, Lv Guangmei ${ }^{3}$, Li $\mathrm{Pin}^{4}$

Affiliations: ${ }^{1}$ Department of Oncology, Nanjing $1^{\text {st }}$ Hospital Affiliated to Nanjing Medical University, Nanjing 210006, China; ${ }^{2}$ Department of Central Lab, Nanjing $1^{\text {st }}$ Hospital Affiliated to Nanjing Medical University, Nanjing 210006, China; ${ }^{3}$ Department of Medicine and Nursing Education, Proffessional College of Jiangsu Jiankang, Nanjing 210029, China; ${ }^{4}$ Department of Gastroenterology, Changhai Hospital, Shanghai 200433, China.

Corresponding Author: $\mathrm{Li}$ Pin, MD, Department of Gastroenterology, Changhai Hospital, Shanghai 200433, P.R. China; Fax: +86-25-84800605' Email: pinli1965@163.com

Received: 11 April 2011

Accepted: 19 August 2011

Published: 30 September 2011
79. $4 \%$, while the sensitivity of CA19-9 was $91.8 \%$ and specificity $84.8 \%$. Statistical analysis showed no difference between the plasma LPA concentration and serum CA19-9 activity. However, alteration of plasma LPA has shown significant correlations with the tumor size, pathological classification, pathological stage, infiltration of capsule cells, surrounding lymph nodes and specific histopathological features. Conclusion: LPA might provide clinical physicians with aditional indicator of diagnosis, metastasis and prognosis, making it a promising biomarker for pancreatic cancer. Our findings suggested that LPA would be a potential target for treatment of pancreatic cancers.

Keywords: Lysophosphatidic acid; CA19-9; Pancreatic cancer; Cancer diagnosis

$$
* * * * * * * * *
$$

Yongling G, Shaokai W, Chenjie T, Jinfei C, Shukui W, Xiufeng C, Guangmei L, Pin L. Clinical evaluation on the determination of plasma lysophosphatidic acid concentration in Chinese human pancreatic cancer. International Journal of Hepatobiliary and Pancreatic Diseases 2011;1:6-12.

Article ID: 100002IJHPDGY2011

$$
* * * * * * * * *
$$

doi:10.54348/ijhpd-2011-2-OA-2

\section{INTRODUCTION}

Pancreatic cancer is one of the most common malignant tumors of human digestive system, and like malignancies of the other parts, it is a type of genetic disease in essence. Its initiation and progression is 
closely related to the excessive proliferation and uncontrolled apoptosis of the tumor cells [1]. Clinical manifestations in middle or advanced stage when discovered are accompanied by local invasion and distant metastasis [2]. The treatment is disappointing and the prognosis is poor. In China, the incidence of pancreatic cancer seems to be increasing. Therefore, early diagnosis and early treatment is of significance to prolong the survival time and improve the quality of life.

The pathogenesis of cancer is characterized by changes in cell growth, survival, differentiation and attachment, leading to uncontrolled tumor growth, invasiveness and metastasis. Lysophosphatidic acid (LPA) has been shown to modulate invasion and growth of tumor cells [3]. Pancreatic ductal adenocarcinoma showed LPA induced cellular responses including cell proliferation and motility [4].

LPA is a naturally occurring phospholipid with diverse effects on various cells. It is widely distributed in many tissues. Like many other biomediators, LPA interacts with cells through specific cell surface receptors ( $\mathrm{G}$ protein-coupled receptors) to induce biological effects. LPA1/Edg-2 (Edg, endothelial differentiation gene), LPA2/Edg-4 and LPA3/Edg-7, named as Edg/LP (lysophospholipid) subfamily, are three most common lysophosphatidic acid receptors. In many sorts of human malignant tumor, there exist metabolic enzymes called autotaxin/lysophospholipid, which is the key enzyme of LPA synthesis. LPA mediated signal transduction through Edg/LPA receptor induces tumor cell proliferation, adhesion, migration, antiapoptotic function and invasion and metastasis, suggesting a common path in carcinogenesis of various human malignancies [5]. In addition, LPA elicited upregulation of VEGF promotes angiogenesis, causing an indirect influence on initiation and progression of malignancies. LPA could stimulate secretion of matrix metalloproteinases (MMPs) and tumor angiogenesis factor, which are closely related to local invasion and metastasis of tumor [6]. Our previous study suggested that MMPs and platelet derived growth factor (PDGF) participated in the process of invasion and metastasis in human pancreatic cancer. The process of tumor initiation and progression is far more complicated, involved multiple steps and many factors [7]. However, the potential role of LPA in carcinogenesis of human malignancies has received great attention.

In recent years, much attention began to be paid to basic and clinical research on the role of LPA and its molecular mechanism in malignant tumors. Thus, LPA might have a potential in clinical diagnosis and therapy of pancreatic cancer. It is of significance to show that levels of plasma LPA and LPA induced biological reactions are associated with the invasive and metastatic pancreatic ductal adenocarcinomas.

In this study, we measured plasma LPA concentration in the pancreatic cancer patients and control subjects and examined the association between the levels of plasma LPA and the tumor size, pathological classification, pathological stage, infiltration of capsule cells, surrounding lymphonoids and specific histopathological features, with focus on evaluation of clinical diagnosis and therapy in human pancreatic cancer.

\section{MATERIALS AND METHODS}

Samples: The samples used in this study were residual samples of those obtained for laboratory analyses (for hospitalization and medical checkups). Those specimens were collected between Jan 2009 and Dec 2010 in Nanjing first people's hospital. Written informed consent was obtained for the use of the residual samples from all the participants. The study was approved by the Institutional Research Ethics Committee of the Faculty of Medicine, the Medical University of Nanjing.

Collected pancreatic cancer specimens were classified as ductal carcinoma (39 cases), acinar carcinoma (4 case), and others (7 cases) by histopathologic examination. Among 50 cases (34 men and 16 women) of pancreatic cancer, in 32 cases the cancer was in the head, in 14 cases in the body and tail, and in four cases in the whole pancreas. Age of the pancreatic cancer patients ranged from $32-64$ years with a mean of 52 years. In accordance with the NCCN pancreatic cancer clinical practice guidelines 2010 (Chinese edition), pathological stage was classified as followings: 2 cases in stage I, 6 cases in stage II, 27 cases in stage III and 15 cases in stage IV. In addition, 32 cases of patients with benign pancreatic lesions (15 cases of pancreatitis, 8 cases pancreatic cyst and 9 cases of pancreatic diabetes) and 36 cases of healthy volunteers were set as controls. All fasting blood samples were collected before breakfast in the morning.

Laboratory reagents: LPA assay kits with reagents and filters were obtained from Thaifushi Technology Development Company in Beijing, China. Tumor marker reagents of CA19-9, CEA and AFP were purchased from Roche, the US.

Plasma lysophosphatidic acid measurements: Participants were asked not to take high fat food, alcoholic liquid and anti-clotting drugs for a week before test. The patient was asked to come for blood collection in a fasting state. Three $\mathrm{ml}$ venous blood was drawn early in the morning. The concentration of plasma LPA was determined in strict accordance with the manual protocol of the LPA assay kit. To obtain the plsma samples, whole blood specimens were directly collected into glass tubes with anticoagulants, then the plasma was separated by centrifugation at $800 \mathrm{~g}$ for $10 \mathrm{~min}$. Centrifugal supernatants fluid $(1 \mathrm{ml})$ was absorbed for extraction of phospholipids, separated by filters, added with colorimetrical reagent and then incubated in $90^{\circ} \mathrm{C}$ water for $5 \mathrm{~min}$. Specimens were removed and kept at room temperature for $35 \mathrm{~min}$, and then measured colorimetrically at $636 \mathrm{~nm}$ wavelength. These LPA measurements were performed using a semi-automatic analyser (BG200oB; Langpu High-Technologies, 
Beijing, China). LPA value $>3 \mu \mathrm{mol} / \mathrm{L}$ was set for positive (+) results, and $\leq 3 \mu \mathrm{mol} / \mathrm{L}$ for negtive (-) results.

Tumor marker determination: Two ml peripheral blood sample (simultaneously collected as LPA blood specimen) was obtained for measurements of serum CA19-9, CEA and AFP level. Using automatic biochemical analyser (E170; Roche, the US). Normal reference value were: AFP: 0 - $25 \mu \mathrm{g} / \mathrm{L}, \mathrm{CEA}:<5 \mathrm{ug} / \mathrm{L}$ and CA19-9: <37 U/ml. Value in normal range was set for negtive (-) results, and abnormal for positive (+) results.

Statistical Analyses: Statistical comparisons were performed with the POMS software (POMS 2.0, Computer Resource Center, $4^{\text {th }}$ Military Medical University, Xian, China), using paired student t test and $\chi^{2}$ test to determine the significance of the association between different variables. The level of significance was set at 0.05 .

\section{RESULTS}

\section{Plasma}

lysophosphatidic

acid measurements: By employing a LPA assay kit and a plasma preparation method suitable for LPA measurement, we examined the plasma LPA concentrations in 50 pancreatic cancer, 32 pancreatic benign lesions and 36 healthy subjects. The distribution of the plasma LPA concentrations are shown in table 1, respectively. The mean $\pm \mathrm{SD}$ of the plasma LPA concentration among the 50 pancreatic cancer subjects was $4.10 \pm 2.03 \mu \mathrm{mol} / \mathrm{L}, 3.28 \pm 1.26 \mu \mathrm{mol} / \mathrm{L}$ in benign lesions subjects and $2.27 \pm 1.02 \mu \mathrm{mol} / \mathrm{L}$ in healthy subjects. The LPA concentration was significantly higher in pancreatic cancer patients than in patients with benign lesions and healthy check-up controls ( $\mathrm{p}<$ 0.05).

The LPA concentration was significantly higher in pancreatic cancer patients than in patients with benign disorders and healthy controls ( $\mathrm{p}<0.05)$.

Sensitivity and specificity analyses between the plasma LPA concentration and other parameters: CA19-9, the so-called gastroenterologyassociated antigen, showed 90\% accuracy in diagnosing pancreatic ductal adenocarcinoma. However, statistical analysis showed a strong positive correlation between the plasma LPA concentration and serum CA19-9 activity $(\mathrm{r}=0.907)$. Other tumor marker indicators like AFP or CEA were only slightly correlated or were not correlated with the LPA concentration (table 2).

For diagnosis of pancreatic ductal carcinoma, the sensitivity of LPA was $89.6 \%$ and the specificity was $79.4 \%$, while the sensitivity of CA19-9 was $91.8 \%$ and specificity was $84.8 \%$ (figure 1). Comparison of sensitivity and specificity between LPA and CA19-9 showed no significant difference ( $p>0.05$ ), suggesting that plasma LPA might be a promising biological indicator in the differentional diagnosis of pancreatic cancer. Plasma LPA level, togethor with serum level of CA19-9, CEA and AFP, might be helpful to confirm a
Table 1: Levels of plasma LPA concentration.

\begin{tabular}{lll}
\hline Groups & n & LPA( $\boldsymbol{\mu m o l} / \mathbf{L})$ \\
\hline Pancreatic cancer & 50 & $4.10 \pm 2.03^{*}$ \\
Pancreatic benign lesions & 32 & $3.28 \pm 1.26$ \\
Healthy check-up & 36 & $2.27 \pm 1.02$ \\
\hline
\end{tabular}

Table 2: Case distribution among the Levels of plasma LPA, AFP, CEA and CA19-9.

\begin{tabular}{lllllllll}
\hline Groups (n) & $\begin{array}{l}\text { LPA } \\
+-\end{array}$ & $\begin{array}{l}\text { AFP } \\
+-\end{array}$ & \multicolumn{2}{l}{$\begin{array}{l}\text { CEA } \\
+-\end{array}$} & $\begin{array}{l}\text { CA19-9 } \\
+-\end{array}$ \\
\hline Pancreatic cancer (50) & 43 & 7 & 9 & 41 & 7 & 43 & 45 & 5 \\
Pancreatic benign lesions & 5 & 27 & 1 & 31 & 3 & 29 & 4 & 28 \\
(32) & & & & & & & &
\end{tabular}

differential diagnosis of pancreatic cancer. Much more work of further investigations need to be done to prove this conclusion.

Plasma LPA Concentation between tumor mass size and clinical stage: The 50 cases of pancreatic cancer were divided into two groups according to the diameter of tumor mass sections, group of diameter $\leq 2 \mathrm{~cm}$ and diameter $>2 \mathrm{~cm}$. In accordance to the NCCN pancreatic cancer clinical practice guidelines 2010 (Chinese edition), 2 cases were in stage I, 6 cases in stage II, 27 cases in stage III and 15 cases in stageIV (table 3).

However, significant difference $(\mathrm{p}<0.05)$ was shown in the plasma LPA levels between the two groups. The group of larger mass (diameter $>2 \mathrm{~cm}$ ) shows a higher level of plasma LPA concentrations than the group with a smaller mass (diameter $\leq 2 \mathrm{~cm}$ ). A lower level of plasma LPA concentrations was observed in stage I-II subjects than in stage III -IV ( $\mathrm{p}>0.05, \mathrm{NS})$. The results showed that as pancreatic tumor mass diameter increased, the tumor burden also rose accordingly, reflected elevated level of LPA concentration in tumor tissue and blood circulation. Thus, LPA induced biological effects of proliferation, anti-apoptosis and invasiveness were also enhanced. In addition, with the

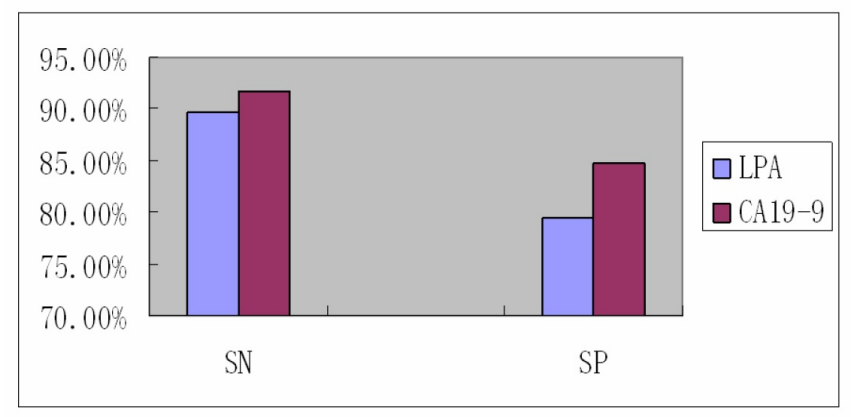

Figure 1: Comparisonf sensitivity and specificity between LPA and CA19-9.

$\mathrm{SN}=\mathrm{TP} /(\mathrm{FP}+\mathrm{TP}) \times 100 \%$; $\mathrm{SP}=\mathrm{TN} /(\mathrm{FN}+\mathrm{TN}) \times 100 \%$.

$\mathrm{SN}$ : sensitivity; SP: specificity; TN: true negative; FN: false negative; TP: true positive; FP: false positive. 
Table 3: Comparisions of plasma LPA between tumor mass diameter and clinical pathological stage.

\begin{tabular}{|c|c|c|c|c|}
\hline Tumor mass diameter & $\frac{\text { I }}{\text { LPA }(\mu \mathrm{mol} / \mathrm{L})(\mathrm{n})}$ & $\frac{\text { II }}{\operatorname{LPA}(\mu \mathrm{mol} / \mathrm{L})(\mathrm{n})}$ & $\frac{\text { III }}{\operatorname{LPA}(\mu \mathrm{mol} / \mathrm{L})(\mathrm{n})}$ & $\frac{\text { IV }}{\text { LPA }(\mu \mathrm{mol} / \mathrm{L})(\mathrm{n})}$ \\
\hline$\leq 2 \mathrm{~cm}$ & $3.03 \pm 0.47(2)$ & $3.16 \pm 0.56(5)$ & $3.72 \pm 1.56(10)$ & $4.13 \pm 2.21(5)$ \\
\hline$>2 \mathrm{~cm}$ & $-(0)$ & $3.97(1)$ & $4.14 \pm 1.85(17)$ & $4.64 \pm 2.42(10)$ \\
\hline
\end{tabular}

progression and development of the disease, LPA secreted by tumor cells increased and was released more into blood circulation, promoting invasion and metastasis of pancreatic cancer cells. The plasma LPA level in various pathological stages showed no obvious difference statisticaly, suggesting that local invasion and distant metastasis might occur at earlier pathological stages of pancreatic cancer, which resulted in higher level of plasma LPA concentrations, difficulty in early diagnosis and poor prognosis.

Plasma LPA concentation and clinical pathological classification: Among the 50 cases, there were 39 cases $(78 \%)$ of ductal carcinoma, whereas acinar carcinoma was $8 \%$ and others $14 \%$ (table 4 ). A higher level of plasma LPA was observed in ductal carcinoma and a lower level of plasma LPA in acinar carcinoma and other pathologial pancreatic cancers. Concentration of plasm LPA in the cases of ductal carcinoma with poor differentiation $(4.27 \pm 2.63 \mu \mathrm{mol} / \mathrm{L})$ was ralatively higher than that in the cases with well differentiation $(3.85 \pm 1.37 \mu \mathrm{mol} / \mathrm{L})$.

A significant difference was shown ( $p<0.05)$ suggesting that higher proliferation of tumor cells in poorly differentiated pancreatic duct carcinomas resulted in more secretion of LPA into circulating blood as well. An elevated level of LPA activates G-protein coupled receptors of Edg and induces biological responses. In the mean time, LPA induced invasion and metastasis was observed in those samples with poorly differentiated pancreatic duct carcinoma.

Plasma LPA concentation and invasion and metastasis: Among 50 cases of pancreatic cancer, there were 42 cases of lymphatic invasion and abdominal metastasis with higher level of plasma LPA $(4.16 \pm 1.96 \mu \mathrm{mol} / \mathrm{L})$ whereas 8 cases of no invasion with lower level of plasma LPA $(3.23 \pm 0.53 \mu \mathrm{mol} / \mathrm{L})$ (table 5).

Increased level of plasma LPA in the invasive cases compared with those free of invasion were of statistical significance $(\mathrm{p}<0.05)$. However, plasma LPA has shown significant correlation with the infiltration of capsule cells, surrounding lymph nodes and specific histopathological features.

\section{DISCUSSION}

Carcinoma of the pancreas usually induces symptoms when the tumor is far advanced (pain, weight loss, jaundice). Physical examination shows little. Chemotherapy and radiotherapy are of limited benefit.
Table 4: Comparisions between plasma LPA concentation and clinical pathological classification.

\begin{tabular}{llll}
\hline $\begin{array}{l}\text { Pathological } \\
\text { classification }\end{array}$ & N \% & LPA( $\left.\boldsymbol{\mu m o l}_{\mathbf{L}} \mathbf{L}\right)$ \\
\hline Ductal carcinoma & 39 & $(39 / 50) 78.0$ & $4.09 \pm 2.18$ \\
Well differentiated & 23 & $(16 / 39) 41.0$ & $3.85 \pm 1.37$ \\
Poorly differentiated & 16 & $(23 / 39) 59.0$ & $4.27 \pm 2.63$ \\
Acinar carcinoma & 4 & $(4 / 50) 8.0$ & $3.85 \pm 1.37$ \\
Others & 7 & $(7 / 50) 14.0$ & $3.90 \pm 1.71$ \\
Total & 50 & - & $4.10 \pm 2.03$ \\
\hline
\end{tabular}

Table 5: Comparisions between plasma LPA concentation and invasion and metastasis.

\begin{tabular}{llll}
\hline $\begin{array}{l}\text { Invasion and } \\
\text { Metastasis }\end{array}$ & N (\%) & LPA $(\boldsymbol{\mu m o l} / \mathbf{L})$ \\
\hline Infiltration & 42 & $(84)$ & $4.16 \pm 1.96$ \\
No Infiltration & 8 & $(16)$ & $3.23 \pm 0.53$ \\
Total & 50 & - & $4.10 \pm 2.03$ \\
\hline
\end{tabular}

The prognosis is dismal [8]. The molecular mechanism of local and distant metastasis is uncertain. Pancreatic cancer cell induced LPA may play a potent role in the course of metastasis of malignant tumors with induction of biological effects such as celluar proliferation, survival and migration etc. We inferred that increased level of LPA correlated with the metastasis and poor prognosis in pancreatic duct carcinoma.

LPA is a simplest small molecules lipid of glycero phosphate originated from membrane. Its glycero skeleton with $\mathrm{C}-3$ for phosphate groups and $\mathrm{C}-2$ for hydroxide radical makes LPA hydrophilic. It could be released to excellular fluid after synthesis, playing the role of signal molecules. Sn sites bind, by chemical ligand, long-chain fatty acids of variable length, which shows lipophilic character and induce biological effects [9].

The serum contents of LPA is extremely little. The range of normal value is $1-3 \mu \mathrm{mol} / \mathrm{L}$. LPA of extracellular synthesis is secreted by platelets, fibroblasts and tumor cell etc. and distributed in serum protein bound form in the circulating blood. LPA could be produced by activated platelets from newly generated, membrane associated PA by the action of phospholipase D (PLD) followed by phospholipase A1 (PLA1) or A2 (PLA2) mediated deacylation. However, the bulk of LPA arising from platelet activation results from the sequential cleavage of serum and membrane 
phospholipids to lysophospholipids by PLA1 and PLA2 secreted from platelets, followed by conversion to LPA by lysophospholipase D (LysoPLD) present in the plasma. Therefore, LPA is also named as plateletderived factor and this might be the main path of LPA synthesis. The plasma LysoPLD is autotaxin (ATX), a member of the nucleotide pyrophosphatase and phosphodiesterase family of exo- and ecto-enzymes. The LPA homeostasis is regulated mainly by the LPA synthesizing enzyme ATX and lipid phosphate phosphohydrolases (LPPs) including LPP1/PAP2A, $\mathrm{LPP}_{3} / \mathrm{PAP}_{2} \mathrm{~B}$ and LPP2/PAP2C. LPA is degraded into monoacylglycerol (MAG) and phosphatidic acid (PA) by LPPs. LPPs play a critical role in controlling levels of extracellular LPA or membrane accessible LPA [10].

In addition to platelet, the other factors such as some inflammatory cells, nerve cells, injury cells, endothelial cells and tumor cells, are stimulated to release LPA in autocrine and paracrine pathway with the induction of various biological effects [11].

We collected blood samples of pancreatic cancer patients for the detection of the serum LPA level. Plasma LPA levels between pancreatic cancer patients and controls were compared and the tested data was assessed by statistical analysis. The results showed that plasma LPA levels of pancreatic cancer group differed significantly from those of the control groups, indicating that the abnormal changes of plasma LPA level might be of significance in clinical diagnosis of pancreatic cancer.

In this study, aimed at the evaluation of the plasma LPA concentration in clinical diagnosis, we examined the plasma LPA concentration in 50 pancreatic cancer subjects $(4.10 \pm 2.03 \mu \mathrm{mol} / \mathrm{L})$ (table 1$)$. The LPA concentration was significantly higher in pancreatic cancer subjects than those in benign pancreatic lesions $(3.28 \pm 1.26 \mu \mathrm{mol} / \mathrm{L})$ and healthy subjects $(2.27 \pm 1.02$ $\mu \mathrm{mol} / \mathrm{L}$ ), which most likely suggested that the higher level of plasm PLA might be of significance in clinical diagnosis of pancreatic cancer. Interestingly, observations were basically similar to those made in a previous report of ovarian carcinoma [12]. Although a weak but significant negative correlation between the concentration of plasma LPA and serum CA19-9 was observed (but not CEA and AFP), its clinical significance remains to be solved.

LPA evokes hormone- and growth factor-like responses in almost all type of cells. LPA stimulates $3 \mathrm{H}-$ thymine base insertion and activation of inositol phosphate, intracellular calcium and protein kinase $\mathrm{C}$ [13]. LPA induces a broad range of cellular effects including smooth muscle cell contraction, platelet aggregation, neurite retraction/cell rounding, regulation of cell proliferation, protection from apoptosis, modulation of chemotaxis and transcellular migration. The biological effects of LPA on tumor cells result in promotion of cell proliferation, survival, drug resistance and metastatic migration [14]. LPA also stimulates secretion of vascular endothelial growth factor (VEGF), interleukin IL - 8 and IL - 6, and growth related oncogene Gro-1, which might be important mediaters in cancer development. For example, vascular permeability affected by VEGF is one of the key step of ovarian cancer ascites formation. Ovarian, breast and prostate cancer patients with increased IL-6 and IL-8 levels showed chemotherapeutic resistance and poor prognosis [15]. Therefore, LPA might contribute to the cellular effecs of tumor angiogenesis and cancer cell proliferation, antiapoptotic activation and local invasion and metastasis. The explanation of biological effects also accords with the hypothesis that LPA promotes tumor cell migration.

We showed that pancreatic cancer cells grew expansively $(\leq 2 \mathrm{~cm})$ and capsulized with fibroblast cells. Direct infiltration was involved in paratumor tissues and capsules. However, tumors $>2 \mathrm{~cm}$ showed visceral metastasis and lymphoid invasion. In this study, plasma LPA showed significant correlation with the size of tumor mass. A lower level of plasma PLA concentration was observed in stage I-II subjects than that in stage III -IV ( $p>0.05$, NS) (table 3). The results suggested that the increased level of plasm LPA contributed to the proliferation of the tumor cells. We concluded that the tumor cells developed in a different way in pancreatic ductal adenocarcinoma, different from that of metastasis. Although the size of the tumor mass is much smaller in the early stages, distant metastasis and postoperative recurrence might develop provided that the pathological classification is poor in some poorly differentiated pancreatic ductal carcinomas.

Tumor cells of low differentiation without, capsular infiltration progressed more easily in the early stages. Both capsule infiltration and lymphoid invasion are characteristic signs involved in local invasiveness and distant metastasis in pancreatic ductal adenocarcinoma. This study has shown that higher level of plama LPA in pancreatic duct carcinoma correlated with capsule infiltration and lymphoid invasion. It also showed that pancreatic cancer cells with a low degree of differentiation had a stronger potential for invasion and metastasis which increased concentration of plasma LPA as the disease progressed.

Besides growth and survival-promoting effects, LPA is a potent mitogen, driving random migration, chemotaxis and tumor cell invasion. Interestingly, the major LPA synthesizing enzyme ATX was originally identified from culture supernatants as a tumor cell motility-stimulating factor. Stimulation of cell motility by LPA and ATX could contribute to the metastatic phenotype of cancer cells. So LPA and ATX might stimulate invasion and metastasis of huaman pancreatic cancer cells as well.

With the recognition of Edg/LPA receptor mediated LPA signal transduction, the potential role of LPA in the development of human pancreatic cancer has gradually become the widely investigated hot point in the research field.

LPA was previously found to be a potent inducer of invasion (transcellular migration) in an in vitro system. A novel LPA, composed of cyclic phosphate and cyclopropane-containing hexadecanoic acid (PHYLPA), first isolated from myxoamoebae of Physarum 
polycephalum, and its synthetic derivatives (cLPA) were tested for their ability to inhibit tumor cell invasion and metastasis. Invasion in vitro by human pancreatic adenocarcinoma cells (PSN-1) was also inhibited by PalcLPA. Thus, Pal-cLPA is effective in inhibiting invasion and metastasis of human pancreatic adenocarcinoma cells [16].

The relevance of LPA to normal and abnormal growth control in vivo is further highlighted by recent studies of PAP-transgene mice. In vivo concentration of LPA is indeed a key factor in the regulation of growth and development. Therefore, abnormalities in both LPA metabolism and LPA function may undermine normal control of cell growth and differentiation [17].

By modifying LPA structures, several groups have developed receptor-subtype specific agonist or antagonist of known LPA receptors. Some species of natural LPA, such as cyclic PA, LPA and LPA with polyunsaturated fatty acid or with fatty acid linked to the sn-2 position, act as relatively receptor-selective ligands. However, it is now possible to alter expression of specific LPA receptors in culture through transfection or in vivo through generation of knockout mice. With the help of biological technique, target therapy for treat Edg/LPA receptors could provide novel strategy to human pancreatic cancer.

We concluded that an understanding of LPA level alteration and biological effects might help physicians to assess the metastatic potential and prognosis of individual patients. LPA is a promising biological indicator in the course of invasion and metastasis. The induction of cellular proliferation, survival and migration may play significant roles in human pancreatic cancer.

In adition, altering LPA production or metabolism may provide an additional strategy to control the function of this important molecule and contribute to clinical treatment of human pancreatic cancer.

$* * * * * * * * *$

\section{Author Contributions}

Gong Yongling - Substantial contributions to conception and design, acquisition of data, Drafting the article, revising it critically for important intellectual content, Final approval of the version to be published Wang Shaokai - Substantial contributions to conception and design, analysis and interpretation of data, Drafting the article, revising it critically for important intellectual content, Final approval of the version to be published

Tao Chenjie - Substantial contributions to conception and design, analysis and interpretation of data, Drafting the article, revising it critically for important intellectual content, Final approval of the version to be published Chen Jinfei - Substantial contributions to conception and design, analysis and interpretation of data, Drafting the article, Final approval of the version to be published Wang Shukui - Substantial contributions to conception and design, Drafting the article, revising it critically for important intellectual content, Final approval of the version to be published

Cao Xiufeng - Substantial contributions to conception and design, analysis and interpretation of data, Drafting the article, revising it critically for important intellectual content, Final approval of the version to be published Lv Guangmei - Substantial contributions to conception and design, analysis and interpretation of data, Drafting the article, revising it critically for important intellectual content, Final approval of the version to be published Li Pin - Substantial contributions to conception and design, analysis and interpretation of data, Drafting the article, revising it critically for important intellectual content, Final approval of the version to be published

\section{Guarantor}

The corresponding author is the guarantor of Submission.

\section{Conflict of Interest}

The authors declare no conflict of interest.

\section{Copyright}

(C) Gong Yongling et al. 2011; This article is distributed under the terms of Creative Commons attribution 3.0 License which permits unrestricted use, distribution and reproduction in any means provided the original authors and original publisher are properly credited. (Please see www.ijhpd.com/copyright-policy.php for more information.)

\section{Acknowledgements}

This work was supported in part by a Grant-in-Aid for the international exchange support plan of Jiangsu Provincal Ministry of Health, Chian. We thank Dr Frank Fang for his technical assistance during our visit in Virginia Commonwealth University.

\section{REFERENCES}

1. Bazzi W, Renon M, Vercherat C, Hamze Z, Lacheretz-Bernigaud A, Wang H. MEN1 missense mutations impair sensitization to apoptosis induced by wild-type menin in endocrine pancreatic tumor cells. Gastroenterology 2008;135(5):1698-9.

2. Sultana A, Smith CT, Cunningham D, et al. Metaanalyses of chemotherapy for locally advanced and metastatic pancreatic cancer. J Clin Oncol 2007;25(9):2607-15.

3. Liu S, Umezu-Goto M, Murph M, et al. Expression of autotaxin and lysophosphatidic acid receptors increases mammary tumorigenesis, invasion, and metastases. Cancer Cell 2009;15(6):539-50.

4. Yamada T, Sato K, Komachi $M$, et al. Lysophosphatidic acid (LPA) in malignant ascites stimulates motility of human pancreatic cancer cells through LPA1. J Biol Chem 2004;279(8):6595-5.

5. Lin ME, Herr DR, Chun J. Lysophosphatidic acid (LPA) receptors: signaling properties and disease relevance. Prostaglandins Other Lipid Mediat 2010;91(3-4):130-8. 
6. $\mathrm{Xu} \mathrm{X}$, Prestwich GD. Inhibition of tumor growth and angiogenesis by a lysophosphatidic acid antagonist in an engineered three-dimensional lung cancer xenograft model. Cancer 2010;116(7):1739-50.

7. Gong YL, Xu GM, Huang WD, Chen LB. Expression of matrix metalloproteinases and the tissue inhibitors ofmetalloproteinases and their local invasiveness and metastasis in Chinese human pancreatic cancer. J Surg Oncol 2000;73(2):95-9.

8. Artinyan A, Anaya DA, McKenzie S, Ellenhorn JD, Kim J. Neoadjuvant therapy is associated with improved survival in resectable pancreatic adenocarcinoma. Cancer 2011;117(10):2044-9.

9. Moolenaar WH. Lysophosphatidic acid, a multifunctional phospholipid messenger. J Biol Chem 1995;270:12949-52.

10. Sigal YJ, Mcdermott MI, Morris AJ. Integral membrane lipid phosphatases /phosphotransferases: common structure and diverse functions. Biochem J 2005;92(5):281-93.

11. Goetzl EJ, An S. Diversity of cellular receptors and functions for the lysophospholipid growth factors lysophosphatidic acid and sphingosine 1-phosphate. FASEB J 1998;12:1589-8.

12. Wang H, Chen DZ. [Study on the diagnostic value of plasma lysophosphatidic acid (LPA) level determination in patients with ovarian carcinoma]. $\mathrm{J}$ of Radioimmunology 2008;21(4):355-7.

13. Moolenaar WH, Kruijer W, Tilly BC, Verlaan I, Bierman AJ, de Laat SW. Growth factor-like action of phosphatidic acid. Nature 1986;323:171-3.

14. van Corven EJ, Groenink A, Jalink K, Eichholtz T, Moolenaar WH. Lysophosphatidate-induced cell proliferation: identification and dissection of signaling pathways mediated by $\mathrm{G}$ proteins. Cell 1989;59:45-4.

15. Stahle M, Veit C, Bachfischer U, et al. Giehl K. Mechanisms in LPA-induced tumor cell migration: critical role of phosphorylated ERK. J Cell Sci 2003;116:3835-46.

16. Mukai M, Imamura F, Ayaki M, et al. Inhibition of tumor invasion and metastasis by a novel lysophosphatidic acid (cyclic lpa). Int $\mathrm{J}$ Cancer 1999;81(6):918-22.

17. Arita $\mathrm{Y}$, Ito $\mathrm{T}$, Oono $\mathrm{T}$, Kawabe $\mathrm{K}$, Hisano $\mathrm{T}$, Takayanagi R. Lysophosphatidic acid induced nuclear translocation of nuclear factor-kB in Panc-1 cells by mobilizing cytosolic free calcium. World $\mathrm{J}$ Gastroenterol 2008;14(28):4473-9. 